\title{
PLASMON-ENHANCED ABSORPTION AND PHOTOCURRENT IN ULTRATHIN GaAS SOLAR CELLS WITH METALLIC NANOSTRUCTURES
}

\author{
Katsuaki Tanabe, Keisuke Nakayama and Harry A. Atwater \\ Thomas J. Watson Laboratory of Applied Physics, California Institute of Technology, Pasadena, CA 91125
}

\begin{abstract}
Metallic nanostructures can excite surface plasmons and can dramatically increase the optical path length in thin active photovoltaic layers to enhance overall photoabsorption. This effect has potential for cost and weight reduction with thinned layers and also for efficiency enhancement associated with increased carrier excitation level in the absorber layer.

We have observed short-circuit current and efficiency enhancements under AM1.5G solar spectrum for $\mathrm{GaAs}$ cells with dense arrays of $\mathrm{Ag}$ nanoparticles deposited through porous alumina membrane masks, relative to reference GaAs cells with no metal nanoparticle array. This photocurrent enhancement is attributed to the scattering effects of metal nanoparticles for light incident into photovoltaic layers. A simple optical model representing metal nanoparticle surface plasmon resonances and multi-angle scattering has been developed and well explains the spectral behavior of the experimental photocurrent enhancement.

A novel ultrathin $\mathrm{GaAs}$ cell structure with a metallic back layer has been also developed with a bonding and layer transfer technique. This waveguide-like GaAs cell showed significant enhancements in short-circuit current density and efficiency relative to reference $\mathrm{GaAs}$ cells with an absorbing $\mathrm{GaAs}$ back layer due to a Fabry-Perot resonance in the air/semiconductor/metal heterostructure.
\end{abstract}

\section{INTRODUCTION}

Recently several groups reported improved photocurrent collection for Si solar cells utilizing enhanced absorption in metal nanoparticle arrays. [1-5] No such study however has been done, to the best of our knowledge, for III-V semiconductor compound solar cells even though the same trade-off between absorption length and carrier diffusion length exists also in III-V cells. In this paper we specifically investigate the effect of arrays of subwavelength-size metal particles on GaAs solar cell absorption and photocurrent.

Metal nanoparticles placed on solar cell surfaces can enhance solar collection, owing to their large extinction cross section which is dominated by scattering rather than absorption for appropriately chosen particle sizes. Nanoparticles scatter the incident light into a wide range of angles to increase the optical path length in the absorber layer. This effect has potential for cell cost and weight reduction resulting from use of thinner absorber layers and also for efficiency enhancement associated with increased carrier excitation level.

\section{ULTRATHIN GaAS SOLAR CELLS WITH METAL NANOPARTICLE ARRAYS}

\section{Experimental}

To demonstrate absorption enhancement by metal nanoparticle scattering, 'optically thin' GaAs solar cells (i.e., intentionally designed with absorber layer thickness < absorption length) with a 50nm-thick p-type emitter on top of a $150 \mathrm{~nm}$-thick $\mathrm{n}$-type base were grown by metalorganic chemical vapor deposition. Dense arrays of metal nanoparticles were then deposited directly onto the $\mathrm{Al}_{0.8} \mathrm{Ga}_{0.2}$ As window layer of the $\mathrm{GaAs}$ cells through porous alumina membrane masks by thermal evaporation.

The optically thin GaAs solar cell structures consisted of, from the top to the bottom of the cell structure $300 \mathrm{~nm}$-thick p-type GaAs contact layer ( $\mathrm{Zn}$ doped, $5 \times 10^{18}$ $\mathrm{cm}^{-3}$ ), which was removed by selective chemical etching except at the front metal contacts, $30 \mathrm{~nm}$ p-type $\mathrm{Al}_{0.8} \mathrm{Ga}_{0.2} \mathrm{As}$ window layer $\left(\mathrm{Zn}, 1 \times 10^{18} \mathrm{~cm}^{-3}\right), 50 \mathrm{~nm}$ p-type GaAs emitter layer $\left(\mathrm{Zn}, 4 \times 10^{18} \mathrm{~cm}^{-3}\right), 150 \mathrm{~nm}$ n-type GaAs base layer $\left(\mathrm{Si}, 2 \times 10^{17} \mathrm{~cm}^{-3}\right.$ ), 500nm n-type $\mathrm{Al}_{0.8} \mathrm{Ga}_{0.2} \mathrm{As}$ back surface field layer (Si, $2 \times 10^{18} \mathrm{~cm}^{-3}$ ), 1um n-type GaAs growth buffer layer $\left(\mathrm{Si}, 2 \times 10^{18} \mathrm{~cm}^{-3}\right.$ ) and n-type GaAs (001) substrate ( $\mathrm{Si}, \sim 1 \times 10^{18} \mathrm{~cm}^{-3}$ ).

The $\mathrm{Ag}$ particle diameters ranged from $50-150 \mathrm{~nm}$, and interpartice spacing ranged from $100-300 \mathrm{~nm}$, with particle shape in the form of upright circular cylinders. The Ag nanoparticle dimensions were quite uniform across each cell sample due to the uniformity of the pore size and spacing in the alumina membrane masks, for which pore diameters were carefully controlled during the membrane preparation. The height of the metal nanoparticles was controlled simply with the deposition thickness in the thermal evaporation process. Fig. 1 shows scanning electron microscope (SEM) images of the Ag nanoparticle arrays with a diameter of $60 \mathrm{~nm}$ and heights ranging through $10 \mathrm{~nm}$ to $75 \mathrm{~nm}$ deposited on the GaAs solar cells.

Spectral response measurements for the fabricated "optically-thin" GaAs solar cells with and without metal nanoparticles of $\mathrm{Ag}$ and $\mathrm{Al}$ were taken to determine the effect of metal nanoparticles.

\section{Modeling}

To understand the role of the metal nanoparticles on the GaAs solar cells more thoroughly, a simple optical model to represent the absorption enhancement in the photovoltaic layers with metal nanoparticles on top has been developed as follows. For simplicity, we consider only the GaAs photovoltaic layer neglecting the AIGaAs window layer. (Note that the refractive indices of $\mathrm{GaAs}$ and AIGaAs are similar.)

The absorption fraction for incident light in a GaAs layer with a thickness of $L$ is simply;

$A_{0}(\lambda)=1-\exp (-\alpha(\lambda) L), \quad$ (Eq. 1)

where $\alpha$ is the absorption constant of GaAs and $\lambda$ is the 
wavelength in vacuo. We assume the angular distribution of the light intensity scattered by sub-wavelength sized particles in the quasistatic limit is

$$
I_{s c a} \propto\left(1+\cos ^{2} \theta\right) I_{0}, \text { [6] (Eq. 2) }
$$

where the angle $\theta$ is measured from the forward to the scattered directions. The absorption fraction for the scattered light is;

$$
A_{\theta}(\lambda)=\int_{0}^{\pi / 2} \frac{1+\cos ^{2} \theta}{\int_{0}^{\pi}\left(1+\cos ^{2} \theta\right) d \theta}\left\{1-\exp \left(-\alpha(\lambda) \frac{L}{\cos \theta}\right)\right\} d \theta^{\prime}
$$

(Eq. 3) accounting that the optical path in the GaAs layer is increased from $L$ into $L / \cos \theta$. The total absorption fraction for the GaAs layer with nanoparticles on top with a surface coverage $\xi$ is;

$$
A_{\text {tot }}(\lambda)=\xi Q_{\text {ext }}(\lambda) \eta_{\text {rad }}(\lambda) A_{\theta}(\lambda)+\left(1-\xi Q_{\text {ext }}(\lambda)\right)(1-R(\lambda)) A_{0}(\lambda)
$$

$\left(\xi Q_{e x t}<1\right), \quad(E q .4)$ where $Q_{e x t}$ and $\eta_{\text {rad }}$ are the extinction efficiency factor and the radiation efficiency for the nanoparticles as defined in Ref. 6 and 2, respectively. $\xi$ was 0.4 and 0.3 for the $60 \mathrm{~nm}$ - and $150 \mathrm{~nm}$-diameter cases, respectively, as determined from SEM images. Values for $Q_{\text {ext }}$ and $\eta_{\text {rad }}$ were calculated for oblate ellipsoidal metal particles with a minor axis parallel to the incident light corresponding to the height of the experimental nanoparticles in the quasistatic limit using an effective medium approximation for the complex dielectric function of the surrounding medium. Particularly for Al nanoparticles, $Q_{\text {ext }}$ and $\eta_{\text {rad }}$ were calculated for concentric $\mathrm{Al}-\mathrm{Al}_{2} \mathrm{O}_{3}$ core-shell ellipsoidal particles with an $\mathrm{Al}_{2} \mathrm{O}_{3}$ shell thickness of $4 \mathrm{~nm}$ accounting for surface oxidation of $\mathrm{Al}$ particles in the atmosphere. The reflectivity $\mathrm{R}$ at the air/GaAs interface is also accounted as follows since our GaAs solar cells had no anti-reflective coating or surface structure. Assuming normal incidence of light into a GaAs layer, the wavelength-dependent reflectivity is written as;

$$
R(\lambda) \equiv \frac{I_{r}(\lambda)}{I_{0}(\lambda)}=\left|\frac{N_{2}(\lambda)-N_{1}(\lambda)}{N_{2}(\lambda)+N_{1}(\lambda)}\right|^{2},
$$

where $N$ is the complex refractive index of air or GaAs.

\section{Results and discussion}

Fig. 2 shows the photocurrent spectra for the GaAs solar cells with metal nanoparticles of $\mathrm{Ag}$ and $\mathrm{Al}$ on top. This photocurrent data is normalized by the spectral response of the reference GaAs cell without metal nanoparticles. A photocurrent enhancement of $260 \%$ is seen at $900 \mathrm{~nm}$ for the GaAs cell with $\mathrm{Ag}$ nanoparticles with a diameter of $150 \mathrm{~nm}$, a height of $20 \mathrm{~nm}$ and $30 \%$ surface coverage. The normalized photocurrent $\left(\mathrm{J} / \mathrm{J}_{\text {ref }}\right)$ is seen to be significantly higher for the cells with $150 \mathrm{~nm}$-diameter nanoparticles, relative to those with a $60 \mathrm{~nm}$-diameter for almost entire spectral range, which can be attributed to the considerably higher radiation efficiency, the ratio of the scattering cross-section to the extinction cross-section, for larger metal nanoparticles as discussed in a following section.

The computed normalized absorption spectra for GaAs solar cells with metal nanoparticles are superposed to the experimental normalized photocurrent for the corresponding cells in Fig. 2. This model calculation is in accord with experimental results. Photocurrent peaks are observed at $300 \mathrm{~nm}$ and $900 \mathrm{~nm}$ and dips at $600 \mathrm{~nm}$ for $60 \mathrm{~nm}$-diameter $\mathrm{Ag}$ and $350 \mathrm{~nm}$ for $60 \mathrm{~nm}$-diameter $\mathrm{Al}$. The dip at $600 \mathrm{~nm}$ in the normalized photocurrent for the cell with $60 \mathrm{~nm}$-diameter $\mathrm{Ag}$ particles, presumably due to the surface plasmon resonance in the $\mathrm{Ag}$ particles, is not seen for $150 \mathrm{~nm}$-diameter particles. This result is attributed to the significantly higher $\eta_{\mathrm{rad}}(\sim 0.9)$ for the $150 \mathrm{~nm}$-diameter case than that $(\sim 0.6)$ for the $60 \mathrm{~nm}$-diameter case suppressing the absorption loss in the $\mathrm{Ag}$ nanoparticles, also seen in the modeling result. For both of $\mathrm{Ag}$ and $\mathrm{Al}$, higher photocurrent enhancement at 900 $\mathrm{nm}$ for $150 \mathrm{~nm}$-diameter cases than for $60 \mathrm{~nm}$-diameter cases is reproduced in the modeling, caused mainly by the higher $Q_{\text {ext }}$ for the larger metal nanoparticles.

Based on these investigations, we have obtained enhancements in net photocurrent, not only for longer wavelength range, and efficiency for certain optimized conditions. We however have observed only slight enhancements in photocurrent and efficiency so far because of the thick absorbing GaAs substrate in the present cell structures. Waveguide-like structures with greater refractive index contrast between a thin photovoltaic layer and a low index substrate layer underneath would give much higher photocurrent enhancements and their fabrication is currently underway.
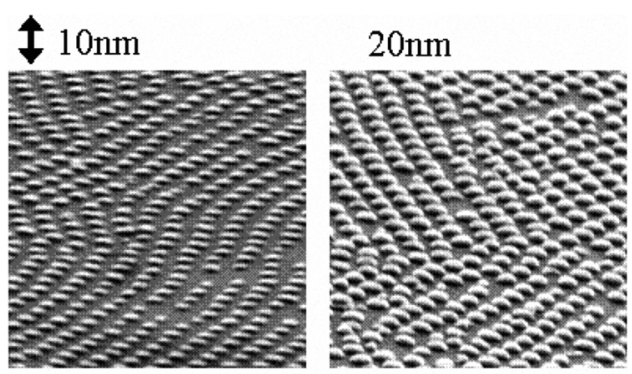

$35 \mathrm{~nm}$
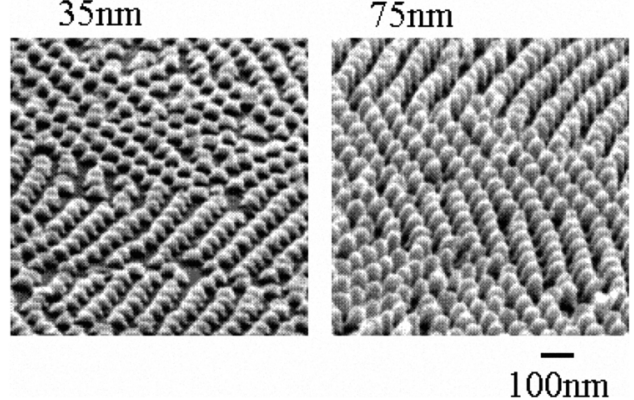

Fig. 1 Scanning electron microscope (SEM) images of the $\mathrm{Ag}$ nanoparticle arrays with a diameter of $60 \mathrm{~nm}$ and heights ranging through $10 \mathrm{~nm}$ to $75 \mathrm{~nm}$ deposited on the $\mathrm{GaAs}$ solar cells with a viewing angle of 75 degree.

\section{ULTRATHIN GaAs SOLAR CELLS WITH METALLIC BACK STRUCTURE}

Experimental

A waveguide-like GaAs solar cell structure with a metal back layer, schematically shown in Fig. 3 (left), was 

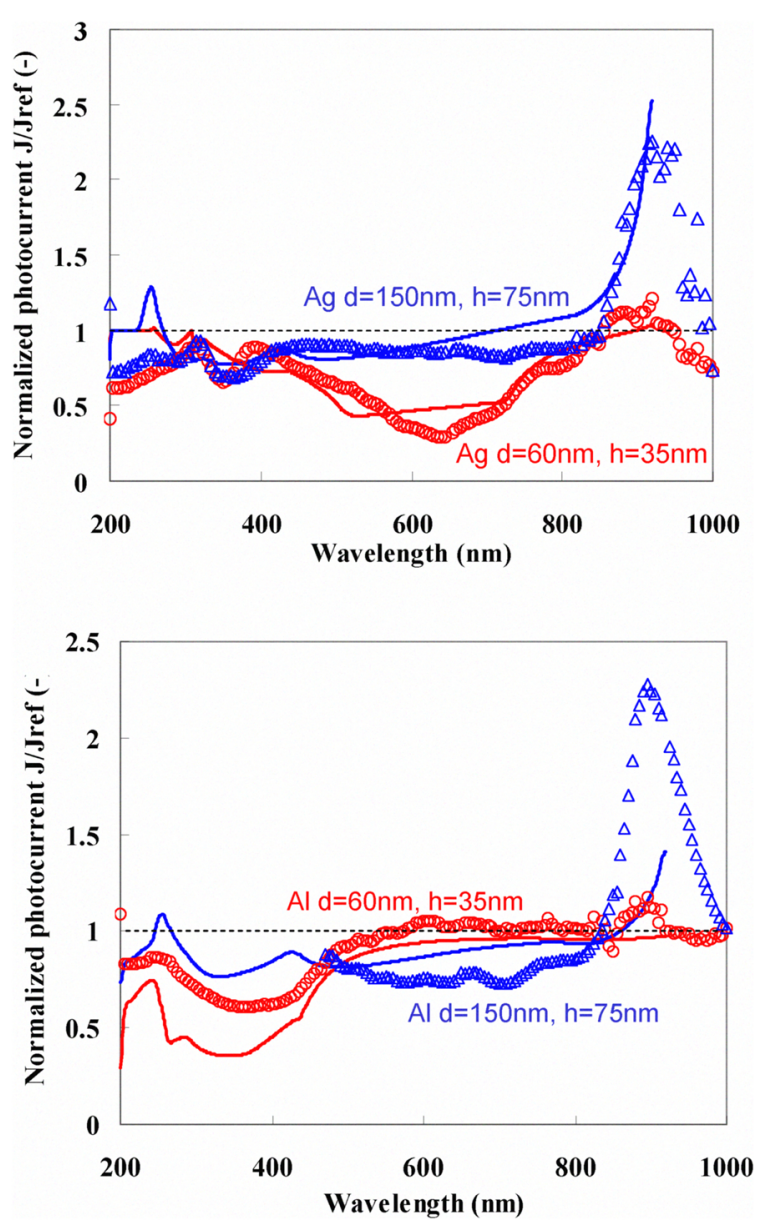

Fig. 2 Normalized photocurrent spectra for the GaAs solar cells with (a) $\mathrm{Ag}$ and (b) Al nanoparticles. Computed normalized absorbance curves in the GaAs solar cells with $\mathrm{Ag}$ and $\mathrm{Al}$ nanoparticles based on the optical model are also plotted.

prepared through wafer bonding and layer transfer with selective etch back of the original GaAs growth substrates. We also fabricated a cell with a $3 \mu \mathrm{m}$ thick GaAs layer, which mimics an absorbing substrate, as a reference shown in Fig. 3 (right). AFM topological images revealed that the GaAs cell / Ag interface had a subwavelength-size roughness, with a peak-valley amplitude around $20 \mathrm{~nm}$, so we expect coupling of the incident light into surface plasmon polariton modes or photonic waveguide modes by multiple angle scattering to enhance absorption.

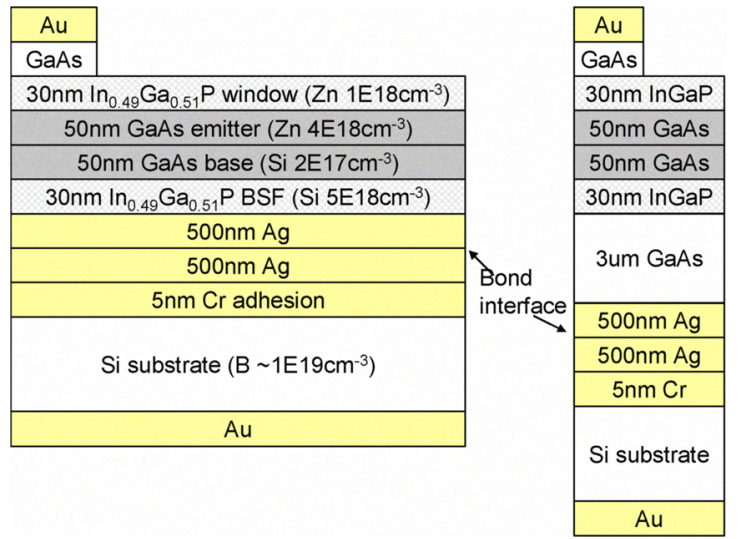

Fig. 3 Schematic cross-sectional diagrams of (left) the waveguide-like GaAs solar cell with metal back layer and (right) the reference cell.

\section{Results and discussion}

Fig. 4 shows the typical light I-V curves for the waveguide-like $\mathrm{GaAs}$ cells with $\mathrm{Ag}$ back structure and the reference $\mathrm{GaAs}$ cells under AM1.5G, 1-sun solar spectrum. The short circuit current is significantly larger for the cells with $\mathrm{Ag}$ back structure. Fig. 5 (triangular dots) shows the normalized photocurrent spectrum, which is the photocurrent of the waveguide-like cell divided by the photocurrent of the reference cell. These light I-V and spectral response results show that the backside-metallized GaAs cell exhibited overall photocurrent enhancement for all wavelengths.

The normalized photocurrent spectrum in Fig. 5 shows two peaks around at $600 \mathrm{~nm}$ and $900 \mathrm{~nm}$. For the dispersion relation at the $\mathrm{GaAs} / \mathrm{Ag}$ interface, the surface plasmon resonance is found at $600 \mathrm{~nm}$, as seen in Fig. 5 . One possible cause of this $600 \mathrm{~nm}$ peak is therefore surface plasmon resonance at the $\mathrm{GaAs} / \mathrm{Ag}$ interface leading to absorption enhancement through incoupling of the incident light into propagating surface plasmon polaritons.

However, optical multilayer calculation of the absorption enhancement indicate a Fabry-Perot effect in the thin active GaAs layer with reflecting back surface shown in Fig. 5 (lines), a peak for the normalized absorbance is also found around at $600 \mathrm{~nm}$ and the two peaks' amplitude ratio is consistent with the calculation result. Also, those two peaks were found to shift as the cell thickness changes consistently with the Fabry-Perot calculation results. Furthermore peaks were found at the same wavelengths for a cell with an Al back layer in spite of the difference for the surface plasmon resonance wavelength between the $\mathrm{GaAs} / \mathrm{Ag}$ and $\mathrm{GaAs} / \mathrm{Al}$ interfaces. For those reasons, the observed photocurrent enhancement for the waveguide-like GaAs cells with metallic back structures characterized by peaks at $600 \mathrm{~nm}$ and $900 \mathrm{~nm}$ is attributed to a Fabry-Perot resonance, and not to surface plasmon polaritons propagation at the GaAs/metal interfaces.

CONCLUSIONS

In this paper two types of plasmonic GaAs solar cell 
were investigated. First, we have observed short-circuit current and efficiency enhancements under AM1.5G solar spectrum for GaAs cells with dense arrays of $\mathrm{Ag}$ nanoparticles deposited through porous alumina membrane masks by thermal evaporation on top of the cell, relative to reference $\mathrm{GaAs}$ cells with no metal nanoparticle array. This photocurrent enhancement is attributed to the scattering effects of metal nanoparticles for light incident into photovoltaic layers. This photocurrent enhancement and the spectral behavior of the normalized photocurrent are qualitatively reproduced by a simple optical model representing surface plasmon resonance of metal nanoparticles and multi-angle scattering.

Second, an ultrathin GaAs cell structure with a metallic back layer has been also developed with a layer transfer technique. This cell showed significant enhancements for short-circuit current and efficiency relative to the reference $\mathrm{GaAs}$ cell with an absorbing $\mathrm{GaAs}$ back layer due to Fabry-Perot resonance in the air/semiconductor/metal heterostructure. Although we have not observed any photocurrent enhancement attributable to propagating surface plasmon polaritons so far, this structure is a good prototype for future exploration of surface plasmon polariton enhanced absorption in solar cells.

\section{ACKNOWLEDGEMENTS}

This work was supported by the DOE, Basic Energy Sciences and GCEP. Dr. Frank Dimroth of the Fraunhofer Institute is acknowledged for the GaAs solar cell growth.

\section{REFERENCES}

[1] H. R. Stuart and D. G. Hall, Appl. Phys. Lett. 69, 1996, pp. 2327-2329.

[2] H. R. Stuart and D. G. Hall, Appl. Phys. Lett. 73, 1998, pp. $3815-3817$.

[3] D. M. Schaadt et al, Appl. Phys. Lett. 86, 2005, pp. 063106-1-063106-3.

[4] S. Pillai et al, Appl. Phys. Lett. 88, 2006, pp. 161102-1-161102-3.

[5] S. Pillai et al, J. Appl. Phys. 101, 2007, pp. 093105-1-161102-8.

[6] C. F. Bohren and D. R. Huffman, Absorption and Scattering of Light by Small Particles (Wiley, New York), 1983, Chap. 3 and 5.

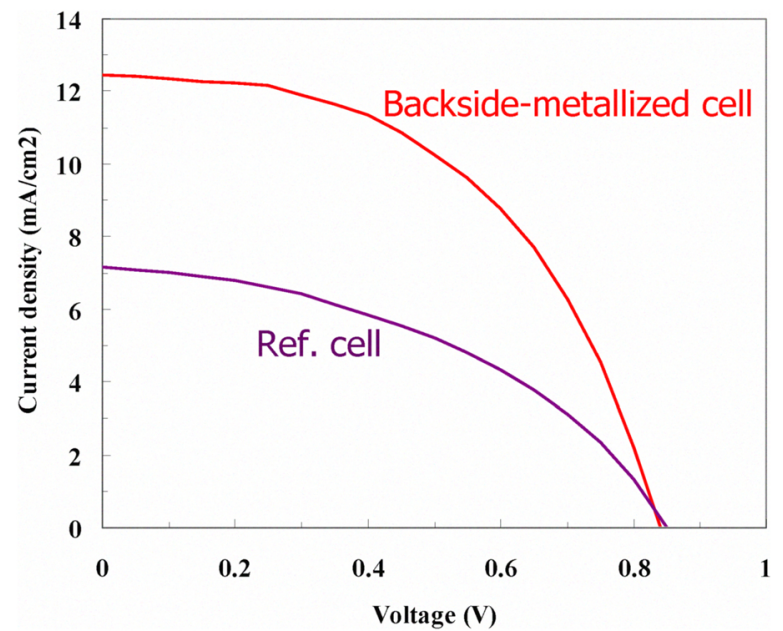

Fig. 4 Typical light I-V curves for the waveguide-like GaAs cells with $\mathrm{Ag}$ back structure and the reference $\mathrm{GaAs}$ cells under AM1.5G 1-sun solar spectrum.

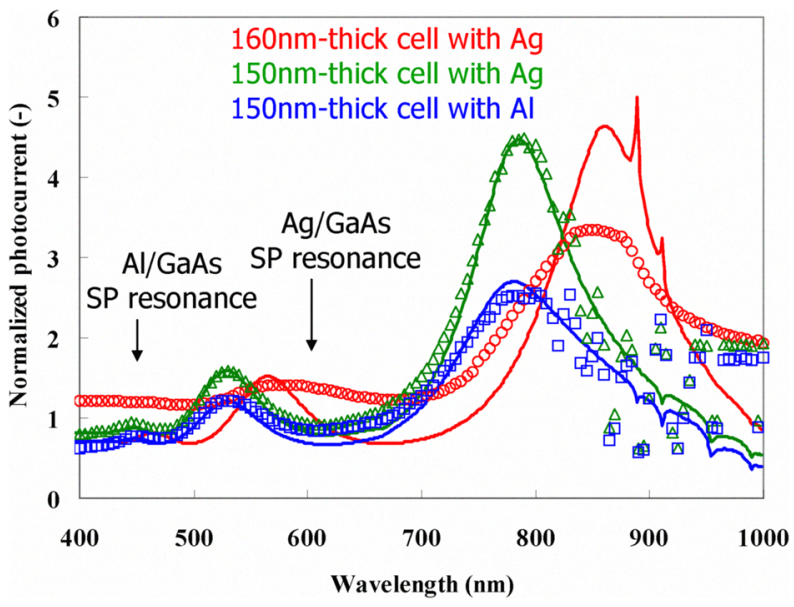

Fig. 5 Normalized photocurrent spectra of the waveguide-like GaAs solar cell relative to the photocurrent of the reference cell. (dots) Calculated normalized absorbance for the waveguide-like GaAs solar cell accounting Fabry-Perot resonance effect in the thin GaAs layer with the metallic back layer and the wavevectors kx parallel to the GaAs/metal interfaces are also plotted. (lines) 